Supporting Information to:

Novel Cadinane and Norcadinane Sesquiterpenes and a New Propanoate from Goldfussia psilostachys

Yinggang Luo

Min Zhou

Huayi Qi

Bogang Li

Guolin Zhang

Affiliation: Chengdu Institute of Biology, the Chinese Academy of Sciences, Chengdu,

P. R. China

Correspondence: Prof. Dr. Guolin Zhang

Chengdu Institute of Biology

Chinese Academy of Sciences

P. O. Box 416

Chengdu 610041

People's Republic of China

Phone/Fax: +86-28-8522-5401

E-mail: zhangg|@cib.ac.cn 


\section{Structure Elucidation of Known Compounds}

2-Ethenyl-2,6,6-trimethyl-3,6-dihydro-2H-pyran (4) [1] was isolated as a natural product for the first time. Lupeol (5) [2], betulin (6) [2], [3], betulinic aldehyde (7) [4], betulinic acid (8) [2], [3], phytol (9) [5], $(22 E, 20 S, 24 R)-5 \alpha, 8 \alpha$-epidioxy-ergosta-6,22-dien-3- $\beta$-ol (10) [6], [7], [8], 5,7-dihydroxy-6,4'-dimethoxyflavone (11) [9], pomolic acid (12) [10], (E)-ferulaldehyde (13) [11], hyptinin (14) [12], 3,5-dimethoxy-4-hydroxybenzaldehyde (15) [13], $\beta$-sitosterol (16) [14], and 2,6-dimethoxy-p-benzoquinone (17) [15], [16], [17] were isolated and identified by their spectral data and optical rotations. $p$-Methoxybenzoic acid (18) and vanillin (19) were identified by co-TLC and co-melting point with authentic samples.

\section{Detailed Extraction and Isolation}

The dried and powdered whole plants of $G$. psilostachys (125 kg) were soaked with $95 \%$ ethanol ( $420 \mathrm{~L} / 7$ days $\times 4$ ) at room temperature. After removing the ethanol under vacuum, ca. $6000 \mathrm{~g}$ of residue were obtained. The syrupy residue was suspended in 7 $L$ water and successively extracted with petroleum ether $\left(60-90^{\circ} \mathrm{C}, 2.4 \mathrm{~L} \times 13\right)$, ethyl acetate $(2.4 \mathrm{~L} \times 13)$ and $n$-butanol $(2.4 \mathrm{~L} \times 13)$ to afford the corresponding extracts $P$ $(1650 \mathrm{~g}), E(100 \mathrm{~g})$ and $B(218 \mathrm{~g})$. The extract $P$ was divided into eighteen fractions $P 1$ - P18 by flash chromatography over silica gel (60 - 100 mesh, $2000 \mathrm{~g})$ eluted with petroleum ether and chloroform (3:1, solvent $A)$ and chloroform and acetone (5:1, solvent B) (each $30,000 \mathrm{~mL}, 15 \mathrm{~mL} / \mathrm{min})$. The fraction P4 (2394 mg, $9000-10,500 \mathrm{~mL}$, solvent A) was subjected to CC over silica gel (200 - 300 mesh, $90 \mathrm{~g}$ ) eluted by cyclohexane and acetone (30:1, $8 \mathrm{~mL} / 15 \mathrm{~min}$ ) to yield compound 9 (40 mg, 850 - 1100 $\mathrm{mL})$. Compounds $5(29 \mathrm{~g}, 120-1350 \mathrm{~mL}), 6(36 \mathrm{~g}, 1500-3800 \mathrm{~mL})$ and 16 (12 g, 5800 - $8400 \mathrm{~mL}$, solvents A) were isolated from P8 (83 g, 20,000 - 22,000 mL) by CC over silica gel (160 - 200 mesh, $500 \mathrm{~g}$ ) eluted by petroleum ether-chloroform-acetone 
(15:10:1, $10 \mathrm{~mL} / \mathrm{min})$. P13 (30 g, 0 - $2000 \mathrm{~mL}$, solvent $\mathrm{B})$ was subjected to CC over $\mathrm{MCl}$ gel $(130 \mathrm{~mL})$ eluted at a rate of $2 \mathrm{~mL} / \mathrm{min}$ to give $\mathrm{P} 13 \mathrm{~A}\left[\mathrm{MeOH}-\mathrm{H}_{2} \mathrm{O}(6: 4)\right.$ as eluent, 0 - $2000 \mathrm{~mL}$ ], P13B [MeOH- $\mathrm{H}_{2} \mathrm{O}(7: 3)$ as eluent, 0 - $2000 \mathrm{~mL}$ ] and P13C [MeOH and $\mathrm{H}_{2} \mathrm{O}(8: 2)$ as eluent, 8500 - 23,500 mL]. Compound 4 (26 mg, $360-470 \mathrm{~mL}$ ) and P13A1 (490 mg, 100 - $200 \mathrm{~mL}$ ) were separated from P13A (610 mg) by CC over silica gel (200 - 300 mesh, $50 \mathrm{~g})$ eluted with chloroform-methanol (50:1, $5 \mathrm{~mL} / 18 \mathrm{~min})$. Compound 18 (14 mg, 400 - $600 \mathrm{~mL}$ ) was isolated from P13A1 by CC over silica gel (200 - 300 mesh, $50 \mathrm{~g})$ eluted with petroleum ether-acetone $(8: 1,0.5 \mathrm{~mL} / \mathrm{min})$. Compound 1 (19 mg) was crystallized from P13B. The precipitate $(800 \mathrm{mg})$ from the chloroform solution of P13C (6700 mg) by adding methanol, was subjected to CC over silica gel (200 - 300 mesh, $70 \mathrm{~g})$ with chloroform-acetone $(40: 1,0.75 \mathrm{~mL} / \mathrm{min})$ as solvent to afford 7 (17 mg, $520-600 \mathrm{~mL})$. The chloroform solution of P13C after removing the precipitate was separated on silica gel (200 - 300 mesh, $300 \mathrm{~g})$ eluted with petroleum ether-acetone $(6: 1,15 \mathrm{~mL} / 8 \mathrm{~min})$ to give P13C6 $(1.4 \mathrm{~g}, 600-720 \mathrm{~mL})$. Compounds 10 (23 mg, $480-570 \mathrm{~mL})$ and 17 (6 mg, $700-780 \mathrm{~mL}$ ) were isolated from P13C6 by CC over silica gel (200 - 300 mesh, 110 g) eluted by petroleum ether-acetone (6:1, $3 \mathrm{~mL} / \mathrm{min})$. P18 (250 g, 12,000 - 17,000 mL, solvent B) was divided into eight fractions (P18A - P18H) by CC over silica gel (160 - 200 mesh, $2000 \mathrm{~g}$ ) eluted with chloroform-acetone (15:1, $50 \mathrm{~mL} / 3 \mathrm{~min})$. P18C (18 g, 4500 - $9500 \mathrm{~mL})$ was subjected to CC over $130 \mathrm{~mL}$ of $\mathrm{MCl}$ gel eluted with methanol-water $(5: 5,5000 \mathrm{~mL}, 6: 4$, $3500 \mathrm{~mL}, 7: 3,7500 \mathrm{~mL}$ and $8: 2,20000 \mathrm{~mL}, 2 \mathrm{~mL} / \mathrm{min}$ ) to give $\mathrm{P} 18 \mathrm{C} 1-4$, respectively. P18C3 (450 mg) was separated on $\mathrm{CC}$ with $50 \mathrm{~g}$ of $\mathrm{C}_{18}$ silica gel eluted with methanol-water (6:4, $4 \mathrm{~mL} / 10 \mathrm{~min})$ to give P18C3A (80 mg, $200-290 \mathrm{~mL})$ and 14 (8 $\mathrm{mg}, 360$ - $390 \mathrm{~mL})$. Compound $2(10 \mathrm{mg}, 110-160 \mathrm{~mL})$ was isolated from P18C3A by CC over silica gel (200 - 300 mesh, $6 \mathrm{~g})$ eluted with chloroform-methanol (30:1, 0.5 $\mathrm{mL} / \mathrm{min}$ ). P18C1 (300 mg) was divided into P18C1A - G by CC over $50 \mathrm{~g}$ of $\mathrm{C}_{18}$ silica gel eluted with methanol-water (4:6, 3 mL/20 min). Compounds 15 (29 mg, 90 - 120 $\mathrm{mL})$ and $19(11 \mathrm{mg}, 50-70 \mathrm{~mL})$ were isolated from P18C1D (57 mg, $150-180 \mathrm{~mL})$ by CC over silica gel (200 - 300 mesh, $5 \mathrm{~g})$ with petroleum ether-acetone $(5: 1,2 \mathrm{~mL} / 3 \mathrm{~min})$ as solvent. P18C1G (24 mg, $210-230 \mathrm{~mL})$ was subjected to CC over silica gel (200 - 
300 mesh, $5 \mathrm{~g}$ ) eluted with petroleum ether-chloroform-acetone $(15: 10: 1,0.5 \mathrm{~mL} / \mathrm{min})$ to afford 13 (8 mg, 80 - $95 \mathrm{~mL}$ ). P18D (7 g, 9500 - 10,300 mL) was divided into P18D1 - 4 by CC (130 $\mathrm{mL}$ of $\mathrm{MCl}$ gel) eluted gradiently with methanol-water $(5: 5,6.5: 3.5$, 7.5:2.5 and 8.5:1.5, $2 \mathrm{~mL} / \mathrm{min}$, each $2000 \mathrm{~mL}$ ). P18D4 (900 mg) was subjected to CC over silica gel ( $200-300$ mesh, $100 \mathrm{~g})$ eluted with petroleum ether-chloroform-acetone (10:5:1, $1 \mathrm{~mL} / \mathrm{min})$ to afford compound 8 (7 mg, 110 - 150 $\mathrm{mL})$. P18E (10 g, 10,300 - 12,800 mL) was separated by CC over $130 \mathrm{~mL}$ of $\mathrm{MCl}$ gel eluted by methanol-water $(6.5: 3.5,1500 \mathrm{~mL}, 5: 5,2000 \mathrm{~mL}, 5.5: 4.5,1500 \mathrm{~mL}$ and 6:4, $2500 \mathrm{~mL}, 2 \mathrm{~mL} / \mathrm{min}$ ) to afford subfractions P18E1 - 5. Compound $11(13 \mathrm{mg}$ ) was precipitated from P18E5 $\left(\mathrm{CH}_{3} \mathrm{OH} / \mathrm{H}_{2} \mathrm{O}, 6: 4,0-2500 \mathrm{~mL}\right)$. P18F $(25 \mathrm{~g}, 12800-17800$ $\mathrm{mL}$ ) was subjected to $\mathrm{CC}$ over $130 \mathrm{~mL}$ of $\mathrm{MCl}$ gel eluted with methanol and water (5:5, $2500 \mathrm{~mL}, 6: 4,5000 \mathrm{~mL}, 7: 3,2500 \mathrm{~mL}$ and $8: 2,5000 \mathrm{~mL}, 2 \mathrm{~mL} / \mathrm{min}$ ) to afford P18F1 - 6 . Compound 12 (22 mg, $210-290 \mathrm{~mL}$ ) was isolated from P18F6 (280 mg, $2500-5000$ $\mathrm{mL}, \mathrm{CH}_{3} \mathrm{OH}-\mathrm{H}_{2} \mathrm{O}, 8: 2$ ) by $\mathrm{CC}$ over silica gel (200 - 300 mesh, $50 \mathrm{~g}$ ) eluted with chloroform-methanol (30:1, $2 \mathrm{~mL} / 3 \mathrm{~min})$. P18H (19 g, 20,800 - 24,300 mL) was divided into $\mathrm{P} 18 \mathrm{H} 1-3$ by $\mathrm{CC}$ on $130 \mathrm{~mL}$ of $\mathrm{MCl}$ gel eluted by methanol-water (6:4, 7:3 and $8: 2$, $2 \mathrm{~mL} / \mathrm{min}$, each $2000 \mathrm{~mL}$ ). Compound 3 (15 mg, $250-320 \mathrm{~mL}$ ) was isolated from $\mathrm{P} 18 \mathrm{H} 1$ (580 mg) by CC over silica gel ( $200-300$ mesh, $50 \mathrm{~g}$ ) eluted with petroleum ether and acetone $(3: 1,1 \mathrm{~mL} / 3 \mathrm{~min})$. All fractions not mentioned above were pigments.

\section{References}

${ }^{1}$ Wu GS, Ma YF, Ge MJ, Li ZL, Li AF, Liu ZJ. Photochemical synthesis of desoxide, nerol oxide and 2-Ethenyl-2,6,6-trimethyl-3,6-dihydro-2H-pyran. Kexue Tongbao 1988; 33: 1319-21 (Chin Sci Bull 1989; 34: 305-8)

${ }^{2}$ Sholichin M, Yamasaki K, Kasai R, Tanaka O. ${ }^{13} \mathrm{C}$ nuclear magnetic resonance of lupane-type triterpenes, lupeol, betulin and betulinic acid. Chem Pharm Bull 1980; 28 : 1006-8

${ }^{3}$ Siddiqui S, Hafeez F, Begum S, Siddiqui SB. Oleanderol, a new pentacyclic triterpene 
from the leaves of Neurium oleander. J Nat Prod 1988; 51: 229-33

${ }^{4}$ Monaco P, Previtera L. Isoprenoids from the leaves of Quercus suber. J Nat Prod $1984 ; 47: 673-6$

${ }^{5}$ Goodman RA, Oldfield E, Allerhand A. Assignments in the natural-abundance carbon-13 nuclear magnetic resonance spectrum of chlorophyll a and a study of segmental motion in neat phytol. J Am Chem Soc 1973; 95: 7553-8

${ }^{6}$ Takashi Y, Uda M. Glycosides of ergosterol from Hericum erinacens. Phytochemistry 1991; 30: 4117-20

7 Sheikh YM, Djerassi C. Steroids from sponges. Tetrahedron 1974; 30: 4095-103

${ }^{8}$ Fisch MH, Ernst R. Identity of ergosterol 5 $3,8 \beta$-peroxide. J Chem Soc Chem Commun 1973; 530

${ }^{9}$ Chari VM, Grayer-Brakmeijer RJ, Harborne JB, Österdahl BG. An acylated allose-containing 8-hydroxyflavone glycoside from Veronica filiformis. Phytochemistry 1981; 20: 1977-9

${ }^{10}$ Cheng DL, Cao XP. Pomolic acid derivatives from the root of Sanguisorba officinalis. Phytochemistry 1992; 31: 1317-20

${ }^{11}$ Barakat $\mathrm{HH}$, Nawwar MAM, Buddrus J, Linscheid M. Niloticol, a phenolic glyceride and two phenolic aldehydes from the roots of Tamarix nilotica. Phytochemistry 1987; 26: $1837-8$

${ }^{12}$ Kuhnt M, Rimpler H, Heinrich M. Lignans and other compounds from the mixe Indian medicinal plant Hyptis verticillata. Phytochemistry 1994; 36: 485-9

${ }^{13}$ Sadtler Research Labortories, Division of Bio-Rad Laboratories, Inc. SADTLER Carbon-13 Nuclear Magnetic Resonance Spectra 44, 1979; 8698C.

${ }^{14}$ Kojima H, Sato N, Hatano A, Ogura H. Sterol glycosides from Prunella vulgaris. Phytochemistry 1990; 29: 2351-5

${ }^{15}$ Bandara BMR, Wimalasiri WR. Diterpene alcohols from Croton lacciferus. Phytochemistry 1988; $27: 225-6$

${ }^{16}$ Handa SS, Kinghorn AD, Cordell GA, Farnsworth NR. Plant anticancer agents. XXVI. Constituents of Peddiea fischeri. J Nat Prod 1983; 46: 248-50

17 Jarvis BB, Pena NB, Cömezóǵlu SN, Rao MM. Non-trichothecenes from Baccharis 
megapotamica. Phytochemistry 1986; 25: 533-5 